\title{
Erratum
}

\section{Erratum: Ueno et al., "Carbon Monoxide, a Retrograde Messenger Generated in Postsynaptic Mushroom Body Neurons, Evokes Noncanonical Dopamine Release”}

In the article "Carbon Monoxide, a Retrograde Messenger Generated in Postsynaptic Mushroom Body Neurons, Evokes Noncanonical Dopamine Release" by Kohei Ueno, Johannes Morstein, Kyoko Ofusa, Shintaro Naganos, Ema Suzuki-Sawano, Saika Minegishi, Samir P. Rezgui, Hiroaki Kitagishi, Brian W. Michel, Christopher J. Chang, Junjiro Horiuchi, and Minoru Saitoe, which appeared on pages 3533-3548 of the April 29, 2020 issue, an acknowledgment for this research was missing. Kohei Ueno was also supported by the Takeda Science Foundation.

The complete funding statement should read as follows:

This work was supported by the Japanese Society for the Promotion of Science Grant JP17K07122 to K.U.; the Takeda Science Foundation to K.U.; Grant-in-Aid for Scientific Research in Innovative Areas “Memory dynamism” JP25115006 to M.S.; and National Institutes of Health Grants GM-79465, ES-28096, and ES-4705 to C.J.C. We thank Tomoyuki Miyashita, Motomi Matsuno, Taro Ueno, and Yukinori Hirano for helpful discussions.

This addition does not affect the conclusion of the article. The article has been updated online.

DOI: 10.1523/JNEUROSCI.1242-20.2020 